\title{
NeuroImage
}

ELSEVIER

www.elsevier.com/locate/ynimg

NeuroImage 27 (2005) $824-834$

\section{The role of precuneus and left inferior frontal cortex during source memory episodic retrieval}

\author{
Brian Nils Lundstrom, ${ }^{\text {a,b }}$ Martin Ingvar, ${ }^{a}$ and Karl Magnus Petersson ${ }^{\text {a,c,d,* }}$ \\ ${ }^{a}$ Cognitive Neurophysiology Research Group, MR Centre, Department of Clinical Neuroscience, Karolinska Institutet, Stockholm, Sweden \\ ${ }^{\mathrm{b}}$ Department of Physiology and Biophysics, University of Washington, Seattle, WA 98195, USA \\ ${ }^{\mathrm{c}}$ Cognitive Neurology and Memory Research Group, F.C. Donders Centre for Cognitive Neuroimaging, University of Nijmegen, The Netherlands \\ ${ }^{\mathrm{d}}$ CSI, Centre for Intelligent Systems, Universidade do Algarve, Faro, Portugal
}

Received 27 October 2004; revised 26 April 2005; accepted 5 May 2005

Available online 27 June 2005

\begin{abstract}
The posterior medial parietal cortex and left prefrontal cortex (PFC) have both been implicated in the recollection of past episodes. In a previous study, we found the posterior precuneus and left lateral inferior frontal cortex to be activated during episodic source memory retrieval. This study further examines the role of posterior precuneal and left prefrontal activation during episodic source memory retrieval using a similar source memory paradigm but with longer latency between encoding and retrieval. Our results suggest that both the precuneus and the left inferior PFC are important for regeneration of rich episodic contextual associations and that the precuneus activates in tandem with the left inferior PFC during correct source retrieval. Further, results suggest that the left ventro-lateral frontal region/ frontal operculum is involved in searching for task-relevant information (BA 47) and subsequent monitoring or scrutiny (BA 44/45) while regions in the dorsal inferior frontal cortex are important for information selection (BA 45/46).

(C) 2005 Elsevier Inc. All rights reserved.
\end{abstract}

Keywords: Episodic memory retrieval; Source memory; Precuneus; Prefrontal cortex; Neuroimaging; Event-related fMRI

\section{Introduction}

Human memory that is important for encoding and retrieving declarative information has been fractionated into working, semantic, and episodic memory (e.g., Eichenbaum and Cohen, 2001; Schacter and Tulving, 1994). Roughly speaking, semantic memory comprises general world knowledge, such as word meaning and word use, and it functions to store, retrieve, and

\footnotetext{
* Corresponding author. Karolinska Institutet, MR Research Center N-8, Department of Clinical Neuroscience, Karolinska Hospital, 17176 Stockholm, Sweden.

E-mail address: karl.magnus.petersson@fcdonders.ru.nl (K.M. Petersson).

Available online on ScienceDirect (www.sciencedirect.com).
}

associate this information to environmental stimuli. On the other hand, working memory refers to the short-term maintenance and manipulation of information during processing. Finally, episodic memory is employed for long-term storage and recall of previous experiences or episodes, and it allows people to reflect upon their personal past. Entailing more than just event memory and event recall, some have suggested that episodic memory necessarily includes a special awareness for subjective time known as autonoetic consciousness; it enables people to mentally travel backwards in time and knowingly retrieve information from a given personal experience (e.g., Baddeley, 2001; Tulving, 2002; Wheeler, 2000). In other words, episodic memory allows current knowledge to be associated with past experiences.

Source memory tasks have commonly been employed to study episodic memory in neuroimaging (e.g., Rugg and Henson, 2002). However, it has been noted that many processes are likely active during memory retrieval, and several recent studies have emphasized the importance of parsing the effects of these processes from each other (Dobbins et al., 2003; Kahn et al., 2004; Rugg et al., 2003). For example, recognition memory can be thought of as comprising at least two processes, recollection and familiarity (e.g., Kelley and Jacoby, 2000; Mandler, 1980; Yonelinas et al., 1996). Recollection involves a detailed recognition that helps associate facts with personal experience, while familiarity entails only a sense of resemblance. Retrieval can also be operationally defined as encompassing multiple processes, such as mode, orientation, success, and effort (Rugg and Wilding, 2000). Retrieval mode is a relatively long-standing state relating to the task at hand, while orientation is a subset of mode that helps determine how a specific retrieval cue, sometimes known as a retrieval probe, will be processed. For example, cues within a task that elicit different kinds of correct answers would then have different orientations, such as between item recognition vs. source memory judgments or cues relating to previously encoded pictures vs. words. Finally, retrieval success relates to the recovery of previously encoded information, while effort relates to task difficulty and is usually assessed by accuracy or reaction times. 
From a previous study of source memory and item recognition (Lundstrom et al., 2003), data suggest that the retrieval of contextual associations, specifically an imagined picture paired with an encoded word, is associated with activation in the posterior precuneus and left prefrontal cortex. Our explanation for the data has been that successful source memory requires the two-step process of context revival and subsequent processing, where the posterior precuneus activates during regeneration of previous contextual associations and the left ventro-lateral prefrontal cortex (PFC) mediates explicit retrieval and integration of the contextual associations (Lundstrom et al., 2003). This is in accord with previous studies of episodic memory retrieval showing precuneual activation (e.g., Cabeza and Nyberg, 2000; Rugg and Henson, 2003) as well as work that has shown the precuneus to be involved in mental imagery recall (e.g., Fletcher et al., 1995; Grasby et al., 1993; Shallice et al., 1994) and retrieval independent of imagery (Krause et al., 1999). Further, neuroimaging studies have noted left-lateralized activation of the PFC and learning-related decreases, i.e., decreased retrieval activation after increased encoding practice, during source memory retrieval tasks (Dobbins et al., 2002; Nolde et al., 1998a; Petersson et al., 2001). However, in our previous study, the left PFC and posterior precuneus were activated in tandem, and it is difficult to determine the specific roles the two areas may play.

In this study, we use the same source memory paradigm as in our previous study (Lundstrom et al., 2003) but increase the latency between encoding and retrieval from $\sim 1$ min to $\sim 3$ days. This increases subject error rates and allows us to make comparisons (e.g., correct source vs. miss and miss vs. new trials) that should, for example, isolate retrieval success from retrieval orientation. From previous experience with this paradigm, we know that subjects effectively encode source memory associations and can subsequently retrieve these associations within minutes with $\sim 88 \%$ accuracy (Lundstrom et al., 2003). Lower accuracy with increased latency is then expected to represent a failure to regenerate and/or retrieve contextual associations rather than a failure to encode them.

The primary purpose of this study is to further examine posterior precuneal and left prefrontal activation during episodic source memory retrieval in order to suggest more specific roles for the posterior precuneus and left PFC. Specifically, we expect activation of the posterior precuneus during comparisons where successful retrieval of relevant information occurs, such as during correct source minus false alarms (as predicted by Gonsalves and Paller, 2000). We also expect that areas in the left ventro-lateral inferior region/frontal operculum will be active when retrieval and integration of information is attempted, such as during correct source, incorrect source, and miss trials. Data from a previous study (Lundstrom et al., 2003) suggested a functional segregation of the left PFC, and we also noted activation of the left ventro-lateral frontal region/frontal operculum during a comparison of source memory old/new effects vs. item recognition old/new effects, suggesting that the area is involved in a process that was more active during source memory than item recognition retrieval, such as information searching and integration. This area has been noted in previous neuroimaging studies of episodic and source memory (e.g., Buckner et al., 1998; Rugg et al., 1999; Takahashi et al., 2002). Similarly, a recent study of world knowledge as well as lexical-semantic integration implicates a left inferior frontal region in the vicinity of Brodmann area 47 (Hagoort et al., 2004).

\section{Materials and methods}

\section{Participants}

Sixteen healthy, right-handed volunteers (13 female) with an average age of 24 years (range $20-30, \mathrm{SD}=2.8$ ) participated in the study. All were pre-screened, had no history of previous neurological conditions, and gave written informed consent. The ethics committee of Karolinska Hospital/Institutet approved the study.

\section{Experimental stimuli and design}

120 common concrete nouns were divided into three 40 -word groups, which were matched for word length (means 4.2-5.1 letters, range 3-8 letters) and frequency (means 56.3-60.0 per million, range $8-482$ per million). Words were selected and paired with corresponding black-and-white line drawing (Snodgrass and Vanderwart, 1980) as in Johansson et al. (2002). A single noun could easily describe each of these drawings, and this label was suitably concrete to evoke a corresponding mental image. Word sets were assigned to encoding and retrieval trial types such that material was counterbalanced temporally as well as with respect to stimulus type.

Volunteers participated in a word-picture association task that required two out-of-scanner encoding sessions and two eventrelated fMRI retrieval sessions (Fig. 1). A latency of 3 days separated encoding and retrieval sessions. During encoding, presented words were followed by either a visually perceived matching picture ("viewed" trial) or by a blank screen prompting subjects to imagine a matching image ("imagined" trial). During retrieval, subjects answered "viewed", "imagined", or "new". For example, an imagined retrieval trial means that the subject had visually perceived the word previously and had imagined a picture with that word.

Encoding and retrieval sessions lasted approximately $8 \mathrm{~min}$ each. Subject responses were recorded via a finger-response pad. The first (thumb), second, and third fingers were used for a 3-point subjective rating during encoding and the three-choice source memory task. All subjects trained outside the scanner before sessions and reported full understanding of the instructions in a post-experiment interview. Blocked encoding consisted of viewed and imagined trial types where "viewed" or "imagined" refers to whether the picture was visually perceived or imagined (Fig. 1). Words were presented for $1 \mathrm{~s}$ followed by $3.5 \mathrm{~s}$ to view or imagine a picture; a fixation cross appeared for $1.5 \mathrm{~s}$ between trials. For viewed trials, an accompanying black-and-white picture followed the presented word; for imagined trials, a blank screen followed the presented word. Subjects were asked to subjectively rate their positive affection toward the viewed or imagined picture on a 3point scale. Encoding trials were presented as 33-s blocks ( 5 trials + $3 \mathrm{~s}$ instructions), which were temporally balanced within sessions and counterbalanced across sessions and subjects. Encoding sessions consisted of two groups of 8 blocks separated by a subject-determined pause of approximately $1 \mathrm{~min}$. After a 3-day latency, event-related retrieval consisted of a source memory task, with the 3 choices of viewed/imagined/new. "Viewed" and 


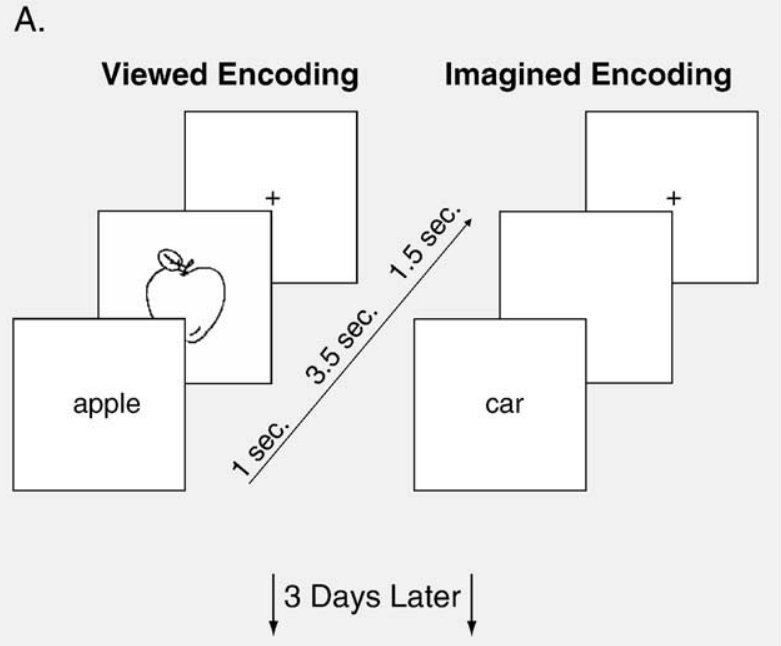

B.

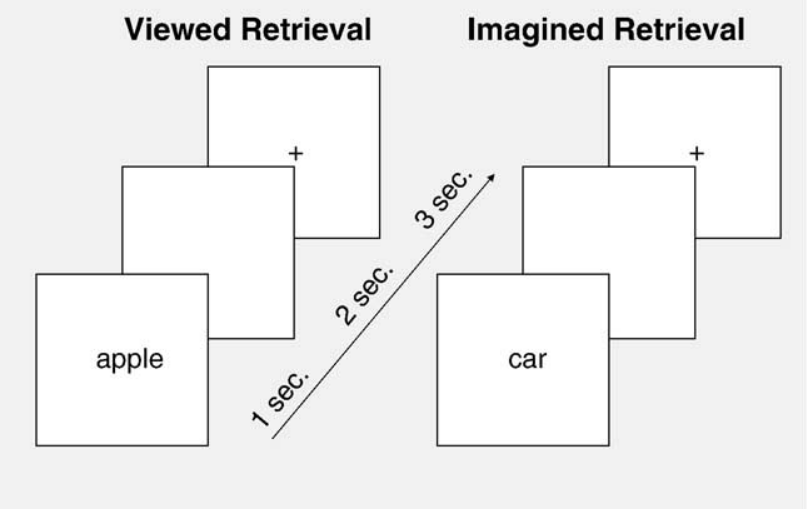

Fig. 1. Experimental design of blocked encoding and event-related retrieval sessions. Encoding and retrieval sessions were separated by a latency of 3 days. (A) Blocked encoding consisted of viewed and imagined trials, where subjects viewed a word and either viewed a corresponding picture or imagined a corresponding mental image. (B) Retrieval consisted of an event-related source memory task.

"Imagined" refer to trials described above, while "New" designates trials in which the presented word was a new, nonencoded word. Words were presented for $1 \mathrm{~s}$ followed by $2 \mathrm{~s}$ of a blank screen. Subjects were required to answer within this 3-s period in a paced paradigm; they were instructed to answer as quickly and accurately as possible but without accidentally responding incorrectly. $3 \mathrm{~s}$ of fixation cross separated every trial. All words were presented a pseudo-random manner such that each of the four trial types (viewed, imagined, new, baseline) were balanced temporally and were presented an equal number of times both midway and at the end of each retrieval section. Each retrieval session was preceded by one 6-s instruction screen and employed 40 old words, 20 new words, and 12 baseline words. For baseline trials, presented words were either Knapp1, Knapp2, or Knapp3 (where "knapp" is translated as button from Swedish). Subjects answered according to the number that followed "knapp".

\section{MRI data acquisition and analysis}

GE Signa 5. $\times$ 1.5-T MR Scanner was employed to obtain T2*weighted echo-planar images (acquisition-matrix $64 \times 64$, voxel
$3.5 \times 3.5 \times 3.4 \mathrm{~mm}$, TE $40 \mathrm{~ms}$, TR $4.2 \mathrm{~s}$ ). 42 slices were acquired in consecutive, ascending order with $3 \mathrm{~mm}$ slice thickness and $0.4 \mathrm{~mm}$ inter-slice spacing. 106 volumes were acquired during each retrieval session. Visual information was presented via back-projection onto a screen, which was viewed by subjects using a binocular-mirror apparatus attached to the head coil.

Image preprocessing was performed using SPM99 software (Wellcome Dept. of Neurology, UK; http://www.fil.ion.ucl.ac.uk). All statistical modeling was performed using the general linear model as implemented in SPM99 (Friston et al., 1995). For each subject, images were realigned to the first volume and resampled using truncated sinc interpolation. Slice timing correction to the middle slice was performed. T1-weighted anatomical image volumes were co-registered to the mean $\mathrm{T} 2 *$-weighted echo planar image (EPI), and spatially normalized to an approximate Talairach space (Talairach and Tournoux, 1988) as defined by the SPM99 MNI T1 template. The transformation parameters thus obtained were applied to the EPI time series, using tri-linear interpolation. Finally, images were spatially filtered using a 10-mm FWHM isotropic Gaussian filter.

Trial lengths were considered to be the time between the onset of word presentation and subject response, i.e., when the subject pressed a button. Every trial type was modeled as an event convolved with the canonical HRF provided by SPM99. Additionally, temporal derivatives of the trial types were modeled as separate regressors. The three kinds of non-baseline events (viewed, imagined, and new) were sorted into correct responses and incorrect responses, which included misses (subject incorrectly answered new), false alarms (subject incorrectly answered viewed or imagined), and correct item recognition but not source recognition. With the inclusion of trial types for event instructions, baseline events, and failure-to-answer (e.g., when the subject did not answer within $3 \mathrm{~s}$ ), nine trial types were modeled separately. Data were high-pass-filtered (range 41-171 s) and low-passfiltered (Gaussian $4 \mathrm{~s}$ FWHM).

Images constituting subject-specific linear combinations of the parameters obtained at the first level, e.g., contrasts spanning parameter estimates for each individual subject, were created. These 16 images, one for every subject, were entered into one second-level model (one-sample $t$ test) for each contrast, yielding a random effects model (Holmes and Friston, 1998). The following comparisons were made: (1) Correct Source (i.e., imagined + viewed) > New; (2) Incorrect Source > New; (3) Correct Source > False Alarms; (4) False Alarms $>$ New; (5) Correct Source > Misses; (6) Misses > New; (7) Incorrect Source > Correct Source; (8) Correct Source $>$ Incorrect Source. Comparisons (1) and (2) are basic "old/new" effects examining source memory and item recognition, respectively. Comparisons (3) through (6) parse the processes involved in successful retrieval; comparisons (3) and (6) isolate the factor of encoded stimulus while comparisons (4) and (5) isolate the factor of subject response. Comparisons (7) and (8) examine directly differences between source and item recognition. In general, we expect increased activity in the posterior precuneus and left PFC during comparisons of increased information retrieval and the increased selection and integration required to retrieve information. Therefore, we expect activity in the posterior precuneus and left PFC to be roughly: Correct Source $\geq$ Incorrect Source $\geq$ False Alarms $\geq$ Misses $\geq$ New. However, since the incorrect source trials comprise successful item recognition in which subjects fail to make the expected source attribution, thereby suggesting increased attempts at information retrieval and scrutiny, 
the comparison of Incorrect Source $\geq$ Correct Source should display left PFC activity. These expectations provide motivation for comparisons (3) through (8).

All inference was based on the SPM $\{t\}$ 's from the second-level analysis. SPM $\{t\}$ 's were height thresholded at the $P<0.001$ uncorrected $[t(15)=3.73]$ level; the number and size of connected clusters of suprathreshold voxels were recorded. Corrected (for multiple comparisons) cluster-level $P$ values were calculated for each cluster based on their spatial extent (Friston et al., 1994). Clusters with a corrected $P$ value $<0.1$, which yielded $\sim 130$ voxels in whole-brain comparisons, were considered significant. Given our interest in the left ventro-lateral frontal cortex/frontal operculum and posterior precuneus, in comparisons (4), (5), and (7) we use a small volume correction of a $20-\mathrm{mm}$ sphere centered at $[-40$,
$20,8]$ as well as one centered at $[-6,-70,36]$ for comparison (5) with both sets of coordinates taken from Lundstrom et al. (2003). To further examine the posterior precuneus at $[-6,-70,36]$, we use a small volume correction testing for the nearest suprathreshold cluster (see MRI results).

\section{Results}

\section{Behavioral performance}

Overall, subjects displayed accuracies that were significantly greater than chance and reaction times that were not significantly different from each other (Table 1). Given random responses,

Table 1

Reaction times and accuracies

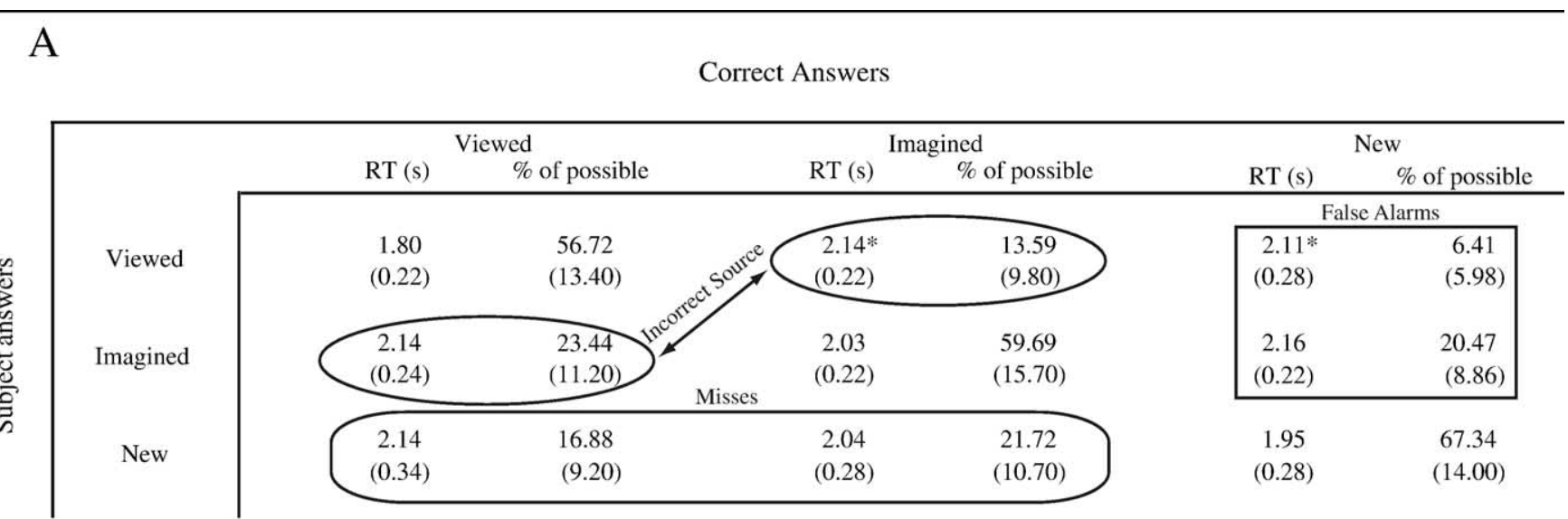

B

Pooled Data

\begin{tabular}{ccc}
\hline Trial type & RT $(\mathrm{s})$ & \% of possible \\
\hline Correct source & 1.92 & 58.20 \\
& $(0.25)$ & $(14.43)$ \\
& 2.14 & 18.52 \\
Incorrect source & $(0.20)$ & $(6.87)$ \\
& 2.08 & 19.30 \\
Misses & $(0.26)$ & $(8.39)$ \\
& & \\
& 2.16 & 26.87 \\
False alarms & $(0.19)$ & $(12.83)$ \\
& & \\
& 1.95 & 67.43 \\
New & $(0.28)$ & $(13.98)$
\end{tabular}

Reaction times (RT) are displayed in milliseconds and accuracies in percentages. Standard deviations are in parentheses. On average, subjects failed to answer in $4.58 \%(7.82 \%)$ of all events $(120$ events total per subject). The asterisk $(*)$ indicates that one subject made no errors of this type. (A) Table displays all possible answers a subject could make. Each of the three columns (Viewed, Imagined, New) contains statistics describing a group of 40 events (words), as detailed in the Methods section. For example, in the "Correct Answers: Viewed" column, the first row "Viewed" displays "56.72\%", which equals $\sim 22.7$ events per subject. (B) These data pertain to the trial types as used for the imaging contrasts. With the exception of "New", the trial types consist of two items from part A. For example, "Incorrect Source" = "Subject Answer: Imagined, Correct Answer: Viewed" + "Subject Answer: Viewed, Correct Answer: Imagined". The percentages of "Correct Source", "Incorrect Source", and "Misses" refer to a total of 80 possible events, while "False Alarms" and "New" refer to a total of 40 possible events. 
accuracies within each of the columns of Table 1A ("Viewed", "Imagined", and "New") would be approximately equal; however, this is not the case for column 1 "Viewed" $[F(2,45)=56.3, P<$ $0.001]$, column 2 "Imagined" $[F(2,45)=63.7, P<0.001]$, or column 3 "New" $[F(2,45)=157.8, P<0.001]$. When subjects made an incorrect source attribution, as was the case during "Incorrect Source" trials and "False Alarm" trials, they displayed a tendency to respond "imagined" rather than "viewed." This is demonstrated by inequalities between the two components of the "incorrect source" accuracies $[t(30)=2.65, P=0.006]$ and the two components of the "false alarm" accuracies $[t(30)=5.26, P<0.001]$.

Regarding reaction times, a two-way ANOVA employing factors of encoding trial (i.e., viewed/imagined/new) and subject response (i.e., viewed/imagined/new) did not demonstrate an effect of encoding trial $[F(2,135)=0.44, P=0.644]$ or of subject response $[F(2,135)=1.68, P=0.191]$ but did display an interaction effect $[F(4,135)=5.63, P<0.001]$. Reaction times for incorrect source trials $[t(30)=3.12, P=0.002]$, false alarm trials $[t(30)=3.06, P=$ $0.002]$, and misses $[t(30)=2.04, P=0.025]$ were longer than for correct source trials. Reaction times for incorrect source $[t(30)=$ 2.33, $P=0.014]$ and false alarm trials $[t(30)=2.21, P=0.017]$ were longer than for new trials, but reaction times between misses and new trials $[t(30)=1.48, P=0.075]$ and between correct source and new trials $[t(30)=0.3471, P=0.367]$ did not differ.

\section{MRI results}

In general, with regard to the posterior parietal regions and prefrontal cortex, data display left prefrontal cortex ( BA 44/45/ 46/47) activations in all comparisons with the exception of correct source minus false alarms and correct source minus incorrect source (Figs. 2 and 3), and posterior precuneal activations in comparisons of correct source trials with false alarms, misses, and new trials (Fig. 4). There was activation in the right PFC in the correct source minus new comparison, and no significant activation was seen in the correct minus incorrect source comparison (Table 2).

In the comparison of incorrect source and new trials, a tripartite activation in the left prefrontal cortex is seen in Fig. 2A with maxima at $[-40,14,8],[-42,30,18]$, and $[-34,24,-2]$ (Table 2). Maxima in similar locations are seen in Figs. $2 \mathrm{~B}-\mathrm{E}$. The maximum in Fig. 2B [-40, 10, 10] is within $5 \mathrm{~mm}$ of $[-40,14,8]$, the maximum in Fig. $2 \mathrm{C}[-42,28,24]$ is within $6 \mathrm{~mm}$ of $[-42,30$, $18]$, and the maxima in Figs. 2D-E ([-40, 20, -2]; [-40, 16, 0]) are within $7 \mathrm{~mm}$ and $10 \mathrm{~mm}[-34,24,-2]$, respectively. Thus, the
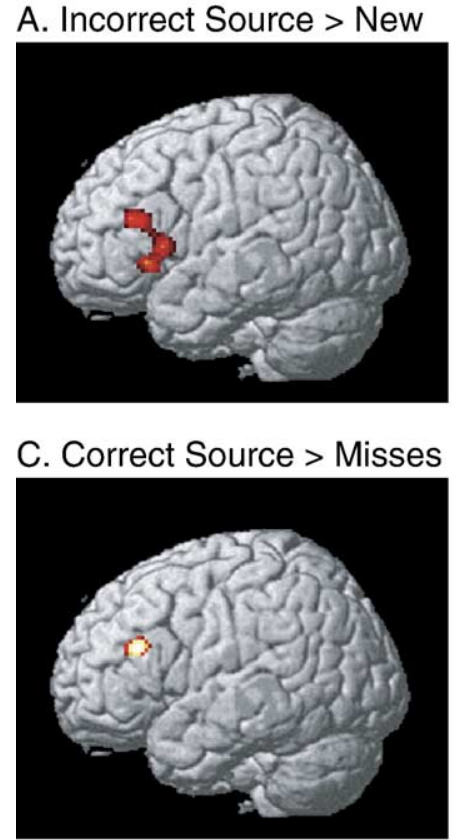

\section{E. False Alarms $>$ New}

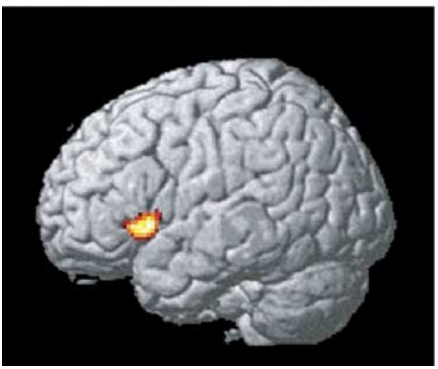

\section{B. Incorrect Source > Correct Source}

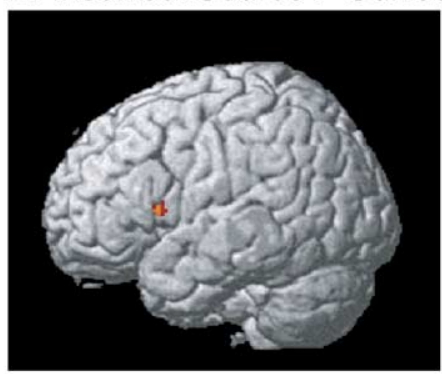

\section{Misses > New}

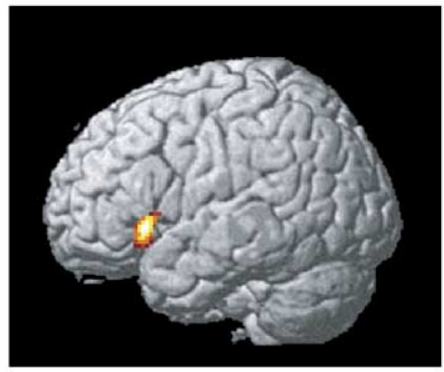

Fig. 2. Activations in the left ventro-lateral frontal region/frontal operculum and left dorsal inferior frontal region. See also Table 2. (A) Incorrect source minus new, BA 44/45/46/47. (B) Incorrect source minus correct source, BA 44/45, maximum voxel at (-40, 10, 10). (C) Correct source minus misses, BA 45/46, maximum voxel at $(-42,28,24)$. (D) Misses minus new, BA 47, maximum voxel at $(-40,20$, -2$)$. (E) False alarms minus new, BA 47, maximum voxel at $(-40,16,0)$. 


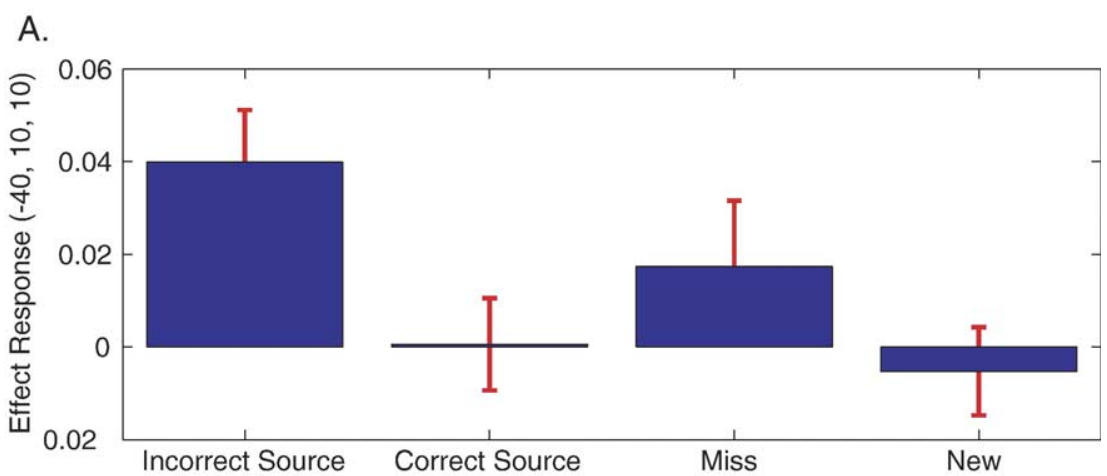

B.

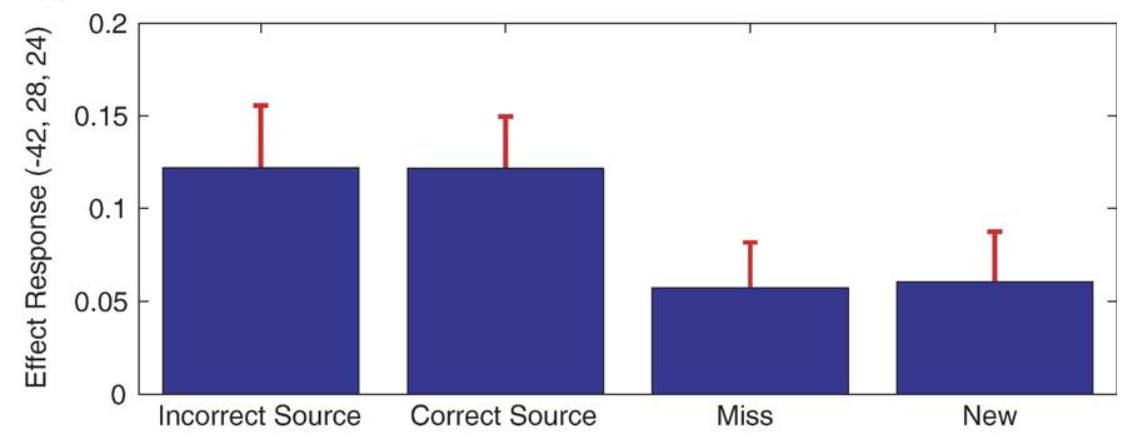

C.

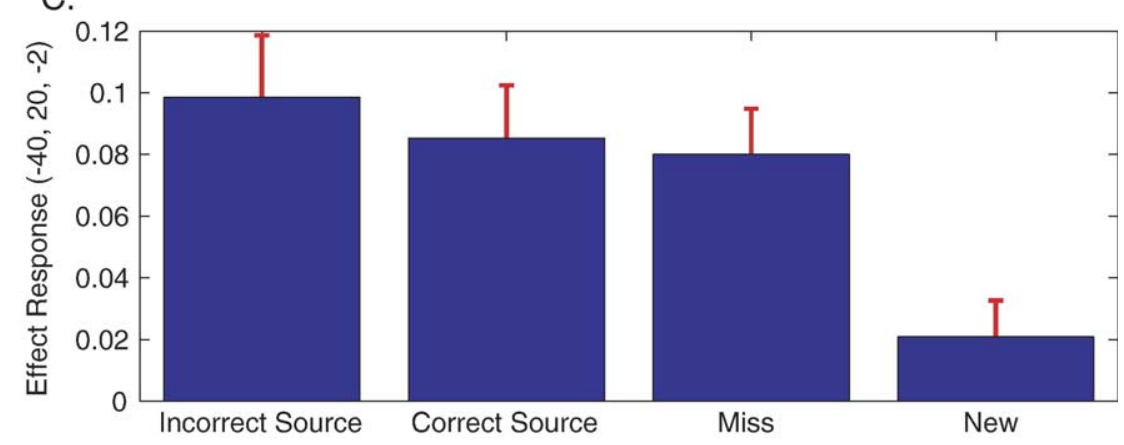

Fig. 3. Parameter estimates for the effect response of three left lateral prefrontal cortex maxima. The rows of these panels (A, B, and C) correspond to the maxima seen in Figs. 2B, C, and D, respectively, and roughly correspond to the tripartite activation seen in Fig. 2A (see Results). Each row displays the parameter estimates of one maximum for each of four trial types as compared to baseline trials. Red bars indicate standard error.

activation seen in Fig. 2A is apparently composed of maxima seen in Figs. 2B-E. Figs. 3A-C display the parameter estimates for maxima seen in Figs. 2B-D. Thus, the maximum voxel of Fig. 2B is relatively active only in the incorrect source trial (Fig. 3A), the maximum voxel of Fig. $2 \mathrm{C}$ is greater in the incorrect and correct source trials (Fig. 3B), and the maximum voxel in Fig. 2D is
A. Correct Source > False Alarms

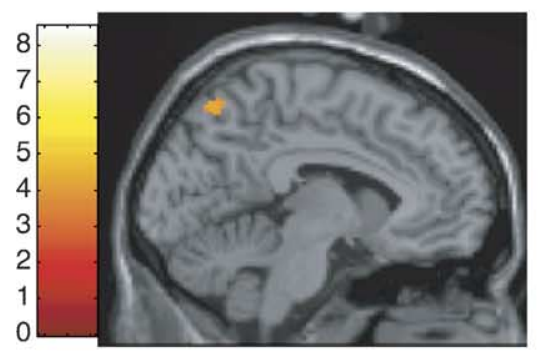

\section{B. Correct Source > Misses}

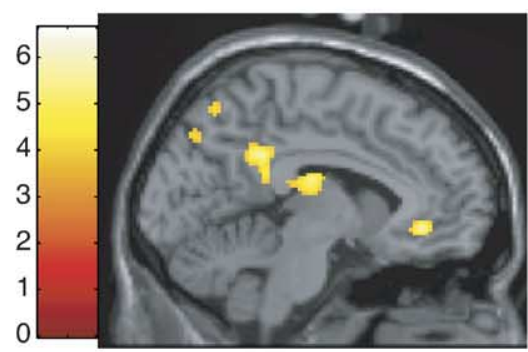

\section{Correct Source > New}

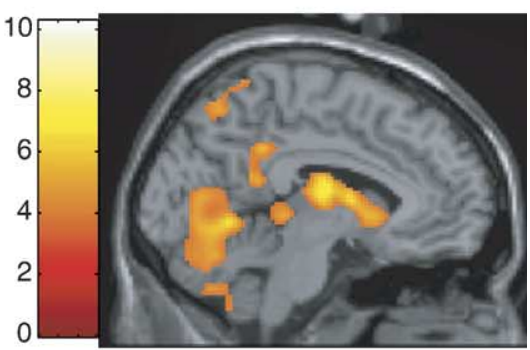

Fig. 4. Posterior precuneal cortex activations thresholded at $P<0.001[t(15)=3.73]$ with an extent greater than $\sim 130$ voxels displayed on sagittal anatomical slices. Color bars reflect the value of the $t$ statistic. (A) Correct source minus false alarms; $x=-6$. (B) Correct source minus misses; $x=-6$. Small volume correction used. (C) Correct source minus new; $x=-6$. 
Table 2

Source memory retrieval activations

\begin{tabular}{|c|c|c|c|c|c|}
\hline & Area & $x, y, z(\mathrm{~mm})$ & Brodmann area & $Z$ score & Cluster size \\
\hline \multirow[t]{19}{*}{ Correct source $>$ new } & \multirow[t]{3}{*}{ Basal ganglia, thalamus, posterior cingulate } & $-12,6,6$ & 23,31 & 5.51 & 3464 \\
\hline & & $14,2,14$ & & 4.64 & \\
\hline & & $-6,-12,14$ & & 4.61 & \\
\hline & \multirow[t]{3}{*}{ Cerebellum, occipital cortex } & $6,-78,-28$ & $17,18,19$ & 4.57 & 2673 \\
\hline & & $-6,-62,-4$ & & 4.36 & \\
\hline & & $0,-58,-26$ & & 4.33 & \\
\hline & $\mathrm{R}$ cerebellum & $38,-68,-32$ & & 4.37 & 491 \\
\hline & \multirow[t]{3}{*}{ L lateral parietal cortex } & $-42,-44,34$ & $7,19,39,40$ & 4.32 & 942 \\
\hline & & $-38,-62,52$ & & 4.04 & \\
\hline & & $-44,-70,36$ & & 3.75 & \\
\hline & \multirow[t]{3}{*}{$\mathrm{L}$ inferior frontal cortex } & $-46,30,18$ & 45,46 & 4.30 & 1019 \\
\hline & & $-50,8,46$ & 8,9 & 3.86 & \\
\hline & & $-40,22,20$ & $44,45,46$ & 3.86 & \\
\hline & \multirow[t]{2}{*}{$\mathrm{L}$ ventro-lateral frontal cortex/frontal opcm } & $-36,20,-6$ & 47 & 4.11 & 268 \\
\hline & & $-24,24,-14$ & 47 & 3.90 & \\
\hline & \multirow[t]{2}{*}{ Posterior precuneus } & $0,-52,72$ & 7 & 3.78 & 152 \\
\hline & & $-6,-66,58$ & 7 & 3.75 & \\
\hline & \multirow[t]{2}{*}{$\mathrm{R}$ inferior frontal and anterior temporal region } & $48,20,-12$ & 38,47 & 3.73 & 175 \\
\hline & & $52,12,-12$ & 38 & 3.58 & \\
\hline \multirow[t]{7}{*}{ Incorrect source $>$ new } & \multirow[t]{3}{*}{ Cingulate gyrus } & $-6,22,46$ & 32 & 4.48 & 792 \\
\hline & & $-6,10,38$ & 24,32 & 4.28 & \\
\hline & & $0,16,36$ & 32 & 4.18 & \\
\hline & \multirow[t]{2}{*}{$\mathrm{L}$ ventro-lateral frontal cortex/frontal opcm } & $-34,24,-2$ & 47 & 4.20 & 354 \\
\hline & & $-40,14,8$ & 44,45 & 3.82 & \\
\hline & L middle-inferior frontal cortex & $-42,30,18$ & 45,46 & 3.81 & \\
\hline & Occipital cortex & $-8,-78,0$ & 17,18 & 3.65 & 183 \\
\hline \multirow[t]{11}{*}{ Correct source $>$ false alarms } & \multirow[t]{2}{*}{ L cerebellum/occipital cortex } & $-40,-70,-14$ & 19 & 5.07 & 173 \\
\hline & & $-20,-74,-22$ & & 3.57 & \\
\hline & \multirow[t]{3}{*}{ L lateral parietal cortex } & $-50,-70,24$ & 39 & 5.02 & 417 \\
\hline & & $-44,-68,34$ & 19,39 & 4.32 & \\
\hline & & $-36,-66,32$ & 40 & 3.83 & \\
\hline & \multirow[t]{3}{*}{$\mathrm{R}$ cerebellum/lateral occipital cx } & $32,-46,-20$ & & 4.22 & 415 \\
\hline & & $36,-46,-32$ & & 3.86 & \\
\hline & & $48,-60,-14$ & 37 & 3.72 & \\
\hline & \multirow[t]{3}{*}{ Posterior precuneus } & $12,-74,54$ & 7 & 3.65 & 185 \\
\hline & & $22,-74,52$ & 7 & 3.43 & \\
\hline & & $-6,-70,60$ & 7 & 3.41 & \\
\hline \multirow[t]{5}{*}{ False alarms $>$ new } & \multirow[t]{3}{*}{ L cerebellum } & $-8,-46,-24$ & & 4.31 & 188 \\
\hline & & $-20,-42,-24$ & & 3.83 & \\
\hline & & $4,-44,-26$ & & 3.23 & \\
\hline & $\mathrm{L}$ ventro-lateral frontal cortex/frontal opcm & $-40,16,0$ & 47 & 4.26 & 248 \\
\hline & $\mathrm{L}$ inferior frontal cortex* & $-48,18,22$ & 44,45 & 3.46 & 50 \\
\hline \multirow[t]{6}{*}{ Correct source $>$ misses } & \multirow[t]{3}{*}{ Thalamus } & $16,-30,12$ & & 4.46 & 527 \\
\hline & & $-4,-16,20$ & & 4.03 & \\
\hline & & $-18,-26,14$ & & 3.18 & \\
\hline & Posterior cingulate & $-4,-40,32$ & 23,31 & 4.13 & 221 \\
\hline & Posterior precuneus* & $-6,-78,42$ & 7 & 3.25 & 12 \\
\hline & L dorsal inferior frontal cortex* & $-42,28,24$ & 45,46 & 3.83 & 58 \\
\hline Misses $>$ new & L ventro-lateral frontal cortex/frontal opcm & $-40,20,-2$ & 47 & 3.94 & 134 \\
\hline Incorrect source $>$ correct source & L ventro-lateral frontal cortex/frontal opcm* & $-40,10,10$ & 44,45 & 3.86 & 60 \\
\hline Correct source $>$ incorrect source & [No significant activation] & & & & \\
\hline
\end{tabular}

Abbreviations: ant, anterior; L, left; opcm, operculum; R, right. The asterisk (*) designates use of small volume correction with 20 mm sphere at $[-6,-70,36]$ or $[-40,20,8]$.

elevated in the incorrect source, correct source, and miss but not new trials (Fig. 3C). Since activation of the maximum voxel in Fig. 3B appears to be related to retrieval of information used for item recognition, we expect this area to be relatively active during false alarm trials, and we test this prediction with a small volume comparison at $[-42,28,24]$ with $10 \mathrm{~mm}$ sphere, which yielded significant activation for false alarms $>$ new (height threshold $P<$ 0.001 , cluster $P=0.029$, corrected, with maximum at $[-48,20,24]$,
$P=0.039$, corrected) but not for correct source $>$ false alarms (height threshold $P<0.05$, uncorrected). Activation in the posterior precuneus can be seen in Figs. 4A, B, and C. Since we are interested in knowing whether activation in the posterior precuneus increases with increased information retrieval, we performed 2 small volume comparisons with height threshold $P<0.001$, uncorrected, testing for the nearest suprathreshold cluster to $[-6,-70,36]$ from Lundstrom et al. (2003), where the peak is chosen on the basis of 
spatial location rather than height or extent (Friston, 1997): (1) correct source $>$ incorrect source: cluster $P=0.023$ (corrected) with local maximum at $[-10,-72,36], P=0.016$ (corrected); (2) incorrect source $>$ miss: cluster $P=0.025$ (corrected) with local maximum at $[-2,-80,46], P=0.016$ (corrected). Given our criteria for significance as stated above, no significant activity was seen in the medial temporal lobe during planned comparisons. No significant activity was seen when comparing correct source vs. incorrect source trials.

Although the paradigm of this study is very similar to a previous study with shorter latency between encoding and retrieval (Lundstrom et al., 2003), a direct comparison of the imaging data between these studies is difficult since event contrasts differ. Nonetheless, qualitatively, these data are consistent. Behavioral performance in comparable trials (i.e., correct viewed, imagined, and new trials) of this study is lower with lower accuracies (mean decrease of $29.5 \%$, $\mathrm{SD}=0.8 \%$ ) and longer reaction times (mean increase of $351 \mathrm{~ms}$, $\mathrm{SD}=117 \mathrm{~ms}$ ) as expected. Trends seen in the first study with $1 \mathrm{~min}$ latency are the same as seen in this 3-day latency study with the exception of the reaction time for correct new trials, which in the previous study were slightly less than that of correct viewed trials in contrast to the current study. Paradigms in these two studies were identical to the subjects, and subject performance in the first study was $\sim 88 \%$ (Lundstrom et al., 2003) and did not rely on recency-effects of short-term memory due to retrograde interval counting by subjects during the 1-min latency (Baddeley, 1995). Therefore, we expect that the lower behavioral performance in this study does not reflect an overall lack of information encoding. Rather, the lower performance may reflect a lack of encoding durability or efficiency during retrieval, aspects related to forgetfulness.

\section{Discussion}

The primary purpose of this study was to further examine the role of posterior precuneal and left prefrontal activation during episodic source memory retrieval using a similar source memory paradigm as in our previous study (Lundstrom et al., 2003) but with an increased latency between encoding and retrieval. Our major finding relates the levels of precuneal and left inferior prefrontal activations to the behavioral outcome of the source memory task. Specifically, activation of the left inferior prefrontal/frontal operculum (BA 47) seems to reflect initiation and an attempt to integrate retrieved information, where one possible interpretation is that information retrieval is dependent on an interaction with the precuneus. Previously, it has been suggested that the posterior precuneus is sensitive to the quality or amount of information retrieved (Lundstrom et al., 2003; Nyberg et al., 2000; Rugg et al., 1998). Here, data suggest that lower levels of precuneal activation correlate with item recognition but not successful source memory whereas higher levels of precuneal activation are related to both correct item recognition as well as adequate source attribution. Precuneal activation thus appears to be related to the amount of information retrieved for both successful item recognition and adequate source attribution and may be related to successful regeneration of previously encoded contextual associations. The precuneus together with the left inferior prefrontal/frontal operculum (BA 47) can be used to distinguish between correct source, incorrect source, and misses. In other words, the precuneus activates in tandem with this area during correct source retrieval, consistent with a suggestion of Lundstrom et al. (2003). Further, results suggest that the left ventro- lateral frontal region/frontal operculum (BA 47) is involved in information integration while regions in the dorsal inferior frontal cortex are important for information selection and monitoring.

\section{Behavioral data}

While reaction times suggest that subjects found trial types to be of approximately equal difficulty, accuracies suggest a tendency to err by replying "imagined" rather than "viewed" (Table 1). Although self-generation has been thought to strengthen contextual associations, it also appears that self-generated information generally contains less perceptual and spatiotemporal information than perceived events (e.g., Marsh et al., 2001). With the increased latency of 3 days, subjects may simply choose the "fuzzier" option of "imagined" rather than the perceptually richer "viewed". Given the similar source accuracies for correct responses, this is likely not a simple effect of encoding differences but rather supports a qualitative difference between the "imagined" and "viewed" retrieval context (compare with the concept of a 'distinctiveness heuristic', e.g., Schacter et al., 2001).

Given the results in Figs. 2-4 and the fact that Figs. 2D and E show activation in approximately the same area, retrieval effort as exhibited by an increased reaction time can only explain results seen in Figs. 2A and B. Since Fig. 2A is composed of a tripartite left inferior PFC activation cluster of activations seen in Figs. 2B$\mathrm{E}$, one can assume that only the activation shared by Figs. 2A and $\mathrm{B}$ at approximately $[-40,10,10]$ can be attributed to increased retrieval-related processing.

\section{Medial posterior parietal cortex}

Activation of the precuneus has been proposed to play a crucial role in the mental imagery of episodic retrieval (e.g., Fletcher et al., 1995; Grasby et al., 1993; Shallice et al., 1994). However, evidence suggests that the functional role of the precuneus may need to be elaborated, and further evidence suggests that the precuneus plays a more general in episodic retrieval and can be functionally disassociated into an anterior and posterior part (Buckner et al., 1996; Fletcher et al., 1998). In addition, posterior precuneal activation has been observed during a paired word associate memory task employing both concrete and abstract nouns as well as two different presentation modalities (auditory or visual), thus suggesting a function in episodic memory that is independent both of the imagery content of words and of differing presentation modalities (Krause et al., 1999). In a previous study (Lundstrom et al., 2003), we found the posterior precuneus to be preferentially activated during source rather than item recognition and during recollection of an imagined rather than a viewed word-picture contextual association. Since the trials in this last comparison had reaction times that were not significantly different, thereby suggesting that retrieval effort did not play a large role in the activation, it seems likely that these activations resulted at least in part from retrieval success.

In this study, we directly test this hypothesis with comparison of correct source minus misses (Fig. 4B) as well as correct source minus false alarm trials (Fig. 4A). We see posterior precuneal activation in response to successful retrieval as well as an area in the lateral posterior parietal region, which has been noted as a hallmark of retrieval success (Konishi et al., 2000; Wheeler and Buckner, 2004). This is in agreement with other findings relating posterior precuneal activation with successful source retrieval (Dobbins et al., 2003) and with true vs. false recognition 
(Gonsalves and Paller, 2000; Slotnick and Schacter, 2004). More specifically, in this study, we find that the activity of the posterior precuneus increases with increased retrieval of relevant information for item recognition as well as for correct source attribution (small volume comparisons show significant activation for correct source $>$ incorrect source and incorrect source > miss; see Results). Further, these data are consistent with a suggestion of Lundstrom et al. (2003) that the precuneus and left inferior PFC are important for regeneration and retrieval of rich episodic contextual associations.

\section{Left prefrontal cortex}

Neuroimaging studies have noted that source memory retrieval yields left-lateralized PFC activation and that learning-related decreases during source memory retrieval are left dominant (Dobbins et al., 2002; Nolde et al., 1998a; Petersson et al., 2001). The left lateral PFC may allow and guide access of semantic knowledge (e.g., Hagoort et al., 2004; Poldrack et al., 1999; Wagner et al., 2001). Other hypotheses suggest that the left lateral PFC can be expected during complex tasks of episodic retrieval and plays a more general role, possibly in maintaining retrieval cues and retrieved information, selection of relevant information, inhibiting irrelevant information, or integration of retrieved information (Fletcher and Henson, 2001; Hagoort et al., 2004; Nolde et al., 1998b; Thompson-Schill et al., 1999). In general, our data suggest a tripartite cluster in the left PFC (Fig. 2A) with two of the clusters in the area of the left ventro-lateral frontal region/frontal operculum and one of the clusters in the left dorsal inferior cortex.

\section{Left ventro-lateral frontal region/frontal operculum}

Activation in the left ventro-lateral frontal region/frontal operculum has been seen previously in neuroimaging recognition tasks (e.g., Buckner et al., 1998; Konishi et al., 2000; Rugg et al., 1999; Wagner et al., 1998) and as a characteristic finding during neuroimaging reading tasks (e.g., Fiez and Petersen, 1998). It has been proposed to play a role in semantic processing (e.g., Bookheimer, 2002; Fiez, 1997) as well as during increased retrieval of contextual information (e.g., Lundstrom et al., 2003; Takahashi et al., 2002).

Recent studies of source memory have noted increased left ventro-lateral frontal region/frontal operculum activation during source failure compared with source recognition (Dobbins et al., 2003) as well as increases in neighboring areas for hits relative to misses, suggesting roles not necessarily linked to retrieval success (Kahn et al., 2004). Our results show activation of this region during a comparison of incorrect source (correct item recognition) with correct source (Fig. 2B), as well as comparisons of misses (Fig. 2D) and false alarms (Fig. 2E) with correctly rejected new trials. Although these maxima are close to one another, the activation in Fig. 2B appears to be distinct from those in Figs. 2D and E.

Concerning the cluster in Fig. 2D (BA 47; [-40, 20, 2]), the maximum voxel displays activity for incorrect source, correct source, and miss but not new trials (Fig. 3C). Since reaction times for correct source and misses were not significantly greater than for new trials (Table 1), it is unlikely that this cluster represents only retrieval effort. It is also doubtful that this area is necessarily linked with failure as previous studies have shown activation during success (e.g., Lundstrom et al., 2003; Rugg et al., 1999, 2003). This region would then be a kind of search engine for task-relevant information, and this would be in accord with previous suggestions by others (Rugg et al., 2003; Takahashi et al., 2002). This could also explain why this region is not engaged during new trials; on one hand, subjects may sense the novelty (lack of 'familiarity') of the truly new word and choose 'new', while on the other hand, when they 'miss' and choose 'new', they depend more on an unsuccessful search for source information, i.e., the precuneus fails to activate.

In addition to being slightly posterior and superior, the cluster in Fig. 2B (BA 44/45; [-40, 10, 10]) displays a relative lack of activity in correct source, miss, and new trials relative to the other regions (Fig. 3A) and thus is sensitive to only incorrect source trials; this area is correlated to unsuccessful retrieval of successfully encoded context, i.e., forgetting the context but not the item. It may be recruited when retrieved contextual information is insufficient to make a correct decision concerning source attribution. In other words, when subjects succeed in item retrieval but are unsure regarding source attribution, this area would facilitate further processing of contextual information. This further processing may involve additional internal specification of retrieval cues and could involve source-specific searching for relevant information. Or, subjects may be more closely scrutinizing retrieved information. In other words, three different processes may be involved: (1) subjects unsuccessfully attempt source attribution; (2) they subsequently monitor and scrutinize more closely previous cues and partially retrieved information; (3) should (2) fail, subjects resort to a default strategy, such as when insufficient ('fuzzy') contextual fragments lead to a bias for choosing imagined over viewed, as indicated by the behavioral data.

\section{Left dorsal inferior frontal region}

The third PFC cluster (Fig. 2C; BA 45/46; [-42, 28, 24]) appears to be activated proportionally to retrieval of information used for item recognition (Fig. 3B). If so, one would expect to see some activation of this area during false alarm trials compared with new trials but no activation when compared with correct source trials. We tested this prediction, and our results show this was the case (small volume comparisons show significant activation for false alarms $>$ new but not for correct source $>$ false alarms; see Results). In other words, this region seems to activate during selection of retrieved information for item recognition, consistent with suggestions by Thompson-Schill et al. (1999).

\section{Conclusion}

These data provide support for the idea that the posterior precuneus is an integral part of successfully completed source memory retrieval and that both the precuneus and the left ventrolateral frontal region/frontal operculum (BA 47) are crucial for correct source retrieval, especially when regeneration of rich episodic contextual associations is important. Further, data suggest three disparate regions in the left lateral prefrontal cortex, where the left ventro-lateral frontal region/frontal operculum is involved in information searching (BA 47) and scrutiny or monitoring (BA 44/ $45)$ while regions in the dorsal inferior frontal cortex are important for information selection (BA 45/46).

\section{Acknowledgments}

Grants from the Swedish Medical Research Council VRK2004-15074-01A, the Karolinska Institutet, the Swedish Medical 
Association, the Knut and Alice Wallenberg Foundation, and the Family Hedlund Foundation supported this work. B.N.L. was supported by 2 T32 GM 07266 Medical Scientist Training Program, National Institute of General Medical Sciences. We thank Guillen Fernandez for comments on an earlier version of the manuscript.

\section{References}

Baddeley, A., 1995. Working memory. In: Gazzaniga, M.S. (Ed.), In The Cognitive Neurosciences. MIT Press, Cambridge, MA, pp. 755-764.

Baddeley, A., 2001. The concept of episodic memory. Philos. Trans. R. Soc. Lond., B Biol Sci. 356, 1345-1350.

Bookheimer, S., 2002. Functional MRI of language: new approaches to understanding the cortical organization of semantic processing. Annu. Rev. Neurosci. 25, 151-188.

Buckner, R.L., Raichle, M.E., Miezin, F.M., Petersen, S.E., 1996. Functional anatomic studies of memory retrieval for auditory words and visual pictures. J. Neurosci. 16, 6219-6235.

Buckner, R.L., Koutstaal, W., Schacter, D.L., Wagner, A.D., Rosen, B.R., 1998. Functional-anatomic study of episodic retrieval using fMRI. I. Retrieval effort versus retrieval success. NeuroImage 7, $151-162$.

Cabeza, R., Nyberg, L., 2000. Imaging cognition II: an empirical review of 275 PET and fMRI studies. J. Cogn. Neurosci. 12, 1-47.

Dobbins, I.G., Foley, H., Schacter, D.L., Wagner, A.D., 2002. Executive control during episodic retrieval: multiple prefrontal processes subserve source memory. Neuron 35, 989-996.

Dobbins, I.G., Rice, H.J., Wagner, A.D., Schacter, D.L., 2003. Memory orientation and success: separable neurocognitive components underlying episodic recognition. Neuropsychologia 41, 318-333.

Eichenbaum, H., Cohen, N.J., 2001. From Conditioning to Conscious Recollection: Memory Systems of the Brain. Oxford Univ., Upper Saddle River, NJ.

Fiez, J.A., 1997. Phonology, semantics, and the role of the left inferior prefrontal cortex. Hum. Brain Mapp. 5, 79-83.

Fiez, J.A., Petersen, S.E., 1998. Neuroimaging studies of word reading. Proc. Natl. Acad. Sci. U. S. A. 95, 914-921.

Fletcher, P.C., Henson, R.N., 2001. Frontal lobes and human memory: insights from functional neuroimaging. Brain 124, 849-881.

Fletcher, P.C., Frith, C.D., Baker, S.C., Shallice, T., Frackowiak, R.S., Dolan, R.J., 1995. The mind's eye-precuneus activation in memoryrelated imagery. NeuroImage 2, 195-200.

Fletcher, P.C., Shallice, T., Frith, C.D., Frackowiak, R.S., Dolan, R.J., 1998. The functional roles of prefrontal cortex in episodic memory. II. Retrieval. Brain 121 (Pt 7), 1249-1256.

Friston, K.J., 1997. Testing for anatomically specified regional effects. Hum. Brain Mapp. 5, 133-136.

Friston, K.J., Worseley, K.J., Frackowiak, R.S.J., Mazziotta, J.C., Evans, A.C., 1994. Assessing the significance of focal activations using their spatial extent. Hum. Brain Mapp. 1, 210-220.

Friston, K.J., Holmes, A.P., Worseley, K.J., Poline, J.B., Frith, C.D., Frackowiak, R.S.J., 1995. Statistical parametric maps in functional imaging: a general linear approach. Hum. Brain Mapp. 2, $189-210$.

Gonsalves, B., Paller, K.A., 2000. Neural events that underlie remembering something that never happened. Nat. Neurosci. 3, 1316-1321.

Grasby, P.M., Frith, C.D., Friston, K.J., Bench, C., Frackowiak, R.S., Dolan, R.J., 1993. Functional mapping of brain areas implicated in auditory-verbal memory function. Brain $116(\mathrm{Pt} \mathrm{1}), 1-20$.

Hagoort, P., Hald, L., Bastiaansen, M., Petersson, K.M., 2004. Integration of word meaning and world knowledge in language comprehension. Science 304, 438-441.

Holmes, A.P., Friston, K.J., 1998. Generalisability, random effects, and population inference. NeuroImage (Suppl. 7), S754.

Johansson, M., Stenberg, G., Lindgren, M., Rosen, I., 2002. Memory for perceived and imagined pictures-An event-related potential study. Neuropsychologia 40, 986-1002.

Kahn, I., Davachi, L., Wagner, A.D., 2004. Functional-neuroanatomic correlates of recollection: implications for models of recognition memory. J. Neurosci. 24, 4172-4180.

Kelley, C.M., Jacoby, L.L., 2000. Recollection and familiarity. In: Tulving, E., Craik, F.I.M. (Eds.), The Oxford Handbook of Memory. Oxford Univ. Press, Oxford, pp. 215-228.

Konishi, S., Wheeler, M.E., Donaldson, D.I., Buckner, R.L., 2000. Neural correlates of episodic retrieval success. NeuroImage 12, $276-286$.

Krause, B.J., Schmidt, D., Mottaghy, F.M., Taylor, J., Halsband, U., Herzog, H., Tellmann, L., Muller-Gartner, H.W., 1999. Episodic retrieval activates the precuneus irrespective of the imagery content of word pair associates. A PET study. Brain 122 (Pt 2), 255-263.

Lundstrom, B.N., Petersson, K.M., Andersson, J., Johansson, M., Fransson, P., Ingvar, M., 2003. Isolating the retrieval of imagined pictures during episodic memory: activation of the left precuneus and left prefrontal cortex. NeuroImage 20, 1934-1943.

Mandler, G., Recognizing, G., 1980. The judgment of previous occurrence. Psychol. Rev. 87, 252-271.

Marsh, E.J., Edelman, G., Bower, G.H., 2001. Demonstrations of a generation effect in context memory. Mem. Cogn. 29, 798-805.

Nolde, S.F., Johnson, M.K., D’Esposito, M., 1998a. Left prefrontal activation during episodic remembering: an event-related fMRI study. NeuroReport 9, 3509-3514.

Nolde, S.F., Johnson, M.K., Raye, C.L., 1998b. The role of prefrontal cortex during tests of episodic memory. Trends Cogn. Sci. 2, 399-406.

Nyberg, L., Persson, J., Habib, R., Tulving, E., McIntosh, A.R., Cabeza, R., Houle, S., 2000. Large scale neurocognitive networks underlying episodic memory. J. Cogn. Neurosci. 12, 163-173.

Petersson, K.M., Sandblom, J., Gisselgard, J., Ingvar, M., 2001. Learning related modulation of functional retrieval networks in man. Scand. J. Psychol. 42, 197-216.

Poldrack, R.A., Wagner, A.D., Prull, M.W., Desmond, J.E., Glover, G.H., Gabrieli, J.D., 1999. Functional specialization for semantic and phonological processing in the left inferior prefrontal cortex. NeuroImage 10, 15-35.

Rugg, M.D., Henson, R.N.A., 2002. Episodic memory retrieval: an eventrelated functional neuroimaging perspective. In: Parker, A., et al., (Eds.), The Cognitive Neuroscience of Memory: Encoding and Retrieval. Psychology Press, New York.

Rugg, M.D., Henson, R.N.A., 2003. Episodic memory retrieval: an (eventrelated) functional neuroimaging perspective. In: Parker, A., et al., (Eds.), The Cognitive Neuroscience of Memory: Encoding and Retrieval. Psychology Press, London.

Rugg, M.D., Wilding, E.L., 2000. Retrieval processing and episodic memory. Trends Cogn. Sci. 4, 108-115.

Rugg, M.D., Fletcher, P.C., Allan, K., Frith, C.D., Frackowiak, R.S.J., Dolan, R.J., 1998. Neural correlates of memory retrieval during recognition memory and cued recall. NeuroImage 8, 262-273.

Rugg, M.D., Fletcher, P.C., Chua, P.M., Dolan, R.J., 1999. The role of the prefrontal cortex in recognition memory and memory for source: an fMRI study. NeuroImage 10, 520-529.

Rugg, M.D., Henson, R.N., Robb, W.G., 2003. Neural correlates of retrieval processing in the prefrontal cortex during recognition and exclusion tasks. Neuropsychologia 41, 40-52.

Schacter, D.L., Tulving, E., 1994. What are the memory systems of 1994 ? Memory Systems 1994. MIT Press, Cambridge, MA, pp. 1-38.

Schacter, D.L., Cendan, D.L., Dodson, C.S., Clifford, E.R., 2001. Retrieval conditions and false recognition: testing the distinctiveness heuristic. Psychon. Bull. Rev. 8, 827-833.

Shallice, T., Fletcher, P., Frith, C.D., Grasby, P., Frackowiak, R.S., Dolan, R.J., 1994. Brain regions associated with acquisition and retrieval of verbal episodic memory. Nature 368, 633-635.

Slotnick, S.D., Schacter, D.L., 2004. A sensory signature that distinguishes true from false memories. Nat. Neurosci. 7, 664-672. 
Snodgrass, J.G., Vanderwart, M., 1980. A standardized set of 260 pictures: norms for name agreement, image agreement, familiarity, and visual complexity. J. Exp. Psychol. Hum. Learn. 6, 174-215.

Takahashi, E., Ohki, K., Miyashita, Y., 2002. The role of the parahippocampal gyrus in source memory for external and internal events. NeuroReport 13, 1951-1956.

Talairach, J., Tournoux, P., 1988. Co-Planar Stereotaxic Atlas of the Human Brain. Thieme, Stuttgart.

Thompson-Schill, S.L., D’Esposito, M., Kan, I.P., 1999. Effects of repetition and competition on activity in left prefrontal cortex during word generation. Neuron 23, 513-522.

Tulving, E., 2002. Episodic memory: from mind to brain. Annu. Rev. Psychol. 53, 1-25.

Wagner, A.D., Desmond, J.E., Glover, G.H., Gabrieli, J.D., 1998. Prefrontal cortex and recognition memory. Functional-MRI evidence for contextdependent retrieval processes. Brain 121 (Pt 10), 1985-2002.

Wagner, A.D., Pare-Blagoev, E.J., Clark, J., Poldrack, R.A., 2001. Recovering meaning: left prefrontal cortex guides controlled semantic retrieval. Neuron 31, 329-338.

Wheeler, M.A., 2000. Episodic memory and autonoetic awareness. In: Tulving, E., Craik, F.I.M. (Eds.), The Oxford Handbook of Memory. Oxford Univ. Press, Oxford, pp. 597-608.

Wheeler, M.E., Buckner, R.L., 2004. Functional-anatomic correlates of remembering and knowing. NeuroImage 21, 1337-1349.

Yonelinas, A.P., Dobbins, I., Szymanski, M.D., Dhaliwal, H.S., King, L., 1996. Signal-detection, threshold, and dual-process models of recognition memory: ROCs and conscious recollection. Conscious Cogn. 5, $418-441$. 